\title{
Publisher Correction: Large-area MRI-compatible epidermal electronic interfaces for prosthetic control and cognitive monitoring
}

Limei Tian, Benjamin Zimmerman (D), Aadeel Akhtar, Ki Jun Yu, Matthew Moore, Jian Wu, Ryan J. Larsen (D), Jung Woo Lee, Jinghua Li, Yuhao Liu, Brian Metzger, Subing Qu, Xiaogang Guo, Kyle E. Mathewson, Jonathan A. Fan, Jesse Cornman, Michael Fatina, Zhaoqian Xie, Yinji Ma, Jue Zhang, Yihui Zhang $\mathbb{D}^{\mathrm{D}}$, Florin Dolcos, Monica Fabiani D, Gabriele Gratton, Timothy Bretl (D), Levi J. Hargrove, Paul V. Braun (D), Yonggang Huang and John A. Rogers (D)

Correction to: Nature Biomedical Engineering https://doi.org/10.1038/s41551-019-0347-x, published online 18 February 2019.

In Fig. 4c of this Article originally published, the bottom $y$ axis was incorrectly labelled as 'MRI-ECG ( $\mu \mathrm{V})$ '; the correct label is 'MRI/ ECG'. In addition, in Fig. 4d, the bottom $y$ axis was incorrectly labelled as 'ECG ( $\mu \mathrm{V})$ '; the correct label is 'ECG ( $\mathrm{mV})$ '. The scale bar units were also incorrectly stated as ' $m V$ ', the correct units are ' $\mu \mathrm{V}$ '. The figure has now been amended accordingly. 